\title{
Synthesis and Characterization of Novel Hybrid Poly(methyl methacrylate)/Iron Nanowires for Potential Hyperthemia Therapy
}

\author{
Huey-Wen Liou ${ }^{1}$, Hong-Ming Lin ${ }^{1 *}$, Yeu-Kuang $\mathrm{Hwu}^{2}$, Wen-Chang Chen ${ }^{3}$, Wei-Jen Liou ${ }^{1}$, Li-Chung \\ Lai $^{4}$, Wei-Syuan Lin ${ }^{1}$, Wen-An Chiou ${ }^{4}$
}

\begin{abstract}
${ }^{1}$ Department of Materials Engineering, Tatung University, DeHui St., Taipei, Taiwan; ${ }^{2}$ Institute of Physics, Academia Sinica, Academia Rd., Nankang, Taipei, Taiwan; ${ }^{3}$ Department of Chemical and Materials Engineering, National Yunlin University of Science and Technology, University Road, Section, Douliou, Yunlin, Taiwan; ${ }^{4}$ NISP Lab, Jeong H. Kim Engineering Building, NanoCenter, University of Maryland, College Park, U.S.A.

Email: ${ }^{*}$ hmlin@ttu.edu.tw
\end{abstract}

Received July 22 2 nd 2010 ; revised August $14^{\text {th }}, 2010$; accepted September $30^{\text {th }}, 2010$.

\begin{abstract}
Externally applied magnetic fields have been used in this study to fabricate bamboo-like iron nanowires with or without a layer of Poly(methyl methacrylate) (PMMA). The hybrid PMMA/Fe nanowires were synthesized via hard X-ray synchrotron radiation polymerization with various treatment parameters. The results of XRD show that an oxide layer formed on the surface of the iron nanowires. The $\mathrm{Fe}_{2} \mathrm{O}_{3}$ and $\mathrm{Fe}_{3} \mathrm{O}_{4}$ phases coexist in the iron nanowires without $X$-ray irradiation. After $\mathrm{X}$-ray irradiation, the $\mathrm{Fe}_{2} \mathrm{O}_{3}$ phase transformed into $\mathrm{Fe}_{3} \mathrm{O}_{4}$, which stabilized the iron nanowires. The results of XAS proved this phase transformation. TGA analysis confirmed the thermal properties and solid contents in these specimens. Their ferromagnetic behaviors were examined by magnetic hysteresis measurement, which indicated that the magnetic and structural properties of the nanowires can be manipulated by irradiation treatment. This may lead to a novel synthesis for iron nanowires that can be used in high thermal efficiency hyperthermia therapy.
\end{abstract}

Keywords: Iron Nanowires, Poly(methyl methacrylate), Hybrid, X-Ray Irradiation, XAS, Magnetic Materials

\section{Introduction}

Recently, several research groups have carried out investigations of the magnetic properties of metal wires. They used different methods to prepare nanowires and microwires. Menon et al. [1], Bandyopadhyay et al. [2] and Zheng et al. [3] fabricated Fe, Co and Ni nanowires, respectively, using electrodeposition into nanoporous anodic alumina templates. Pan et al. synthesized NiCo microwires using a hydrothermal method [4]. A templatefree method that combines chemical reduction and a magnetic field is applied to prepare Ni nanowires [5]. The latter method only needs one step in its preparation and no thermal treatment.

Herein, iron nanowires have been prepared by an externally applied magnetic field. Hybrid Poly(methyl methacrylate) (PMMA)/iron nanowires are synthesized by combining iron nanowires with radiation polymerization of PMMA. Hard X-ray synchrotron radiation can be used to initiate polymerization of PMMA and success- fully produces PMMA beads [6]. This method has two essential characteristics. First, it requires only one step for the preparation of $\mathrm{Fe}$ nanowires with a polymerized PMMA coating by hard X-ray synchrotron radiation. Second, the PMMA/iron nanowires can be successfully synthesized without adding emulsifier. Compared with the usual chemical methods of preparing organic/inorganic hybrid materials, the method presented here is a quick, simple and 'green' process, which may reduce environmental pollution and increase broad, practical applications. Hybrid PMMA/Fe nanowires are intentionally designed to manipulate their magnetic properties for application in magnetic hyperthermia. The needle-sharp nanowires enhance friction heating while an alternative magnetic field is applied that increases the heating efficiency in hyperthermia therapy.

In this study, hybrid PMMA/Fe nanowires are investigated by X-ray diffraction (XRD), scanning electron microscopy (SEM), high resolution transmission electron microscopy (HRTEM), X-ray absorption spectroscopy 
(XAS), thermogravimetry (TGA), and vibrating sample magnetometry (VSM). An XAS spectrum, which is basically the measured X-ray absorption coefficient as a function of the incoming photon energy, is generally divided by the role of multiple scattering photoelectrons into two different regions: X-ray Absorption Near-Edge Structure (XANES) and Extended X-ray Absorption Fine Structure (EXAFS). XANES, also referred as NEXAS (Near Edge X-ray Absorption Fine Structure), can be used to investigate the electronic structure of specific elements, and EXAFS is used mainly to investigate the local atomic structure concerning the type and number of elements as well as the nearest neighbor bond length [7]. In this study, the oxidation states of iron will be examined by XAS to reveal the effects of X-ray irradiation on the formation of iron oxides in iron nanowires.

\section{Experimental}

Methyl methacrylate monomer (MMA, Fluka Chemical Reagent Co.) was purified by reduced pressure distillation to remove the inhibitor before polymerization. Ferric chloride hexahydrate $\left(\mathrm{FeCl}_{3} \cdot 6 \mathrm{H}_{2} \mathrm{O}\right)$ and sodium borohydride $\left(\mathrm{NaBH}_{4}\right)$ were obtained from Sigma-Aldrich, Inc. Polyvinylpyrrolidone (PVP, K-30, average molecular weight: ca. 40,000 g/mol) was obtained from Tokyo Kasei Kogyo Chemical Reagent Co. Nitric acid (reagent ACS 99.5\%), methanol and ethyl alcohol were bought from Wako Pure Chemical Industries. All solutions were prepared with deionized water $(>18 \mathrm{M} \Omega)$.

\subsection{Preparation of Iron Wires and PMMA/Iron Nanowires}

Five specimens of iron nanowires and PMMA/Fe nanowires with various parameters were prepared in this study. The first specimen was fabricated using the standard synthesis procedure for magnetic wires. Iron nanowires, denoted as specimen (I), were prepared using an externally applied magnetic field with an intensity of $0.3 \mathrm{~T}$. Normally, $\mathrm{Fe}$ nanowires are produced in a $0.7 \mathrm{M}$ aqueous solution of $\mathrm{FeCl}_{3} \cdot 6 \mathrm{H}_{2} \mathrm{O}$ at room temperature in a high purity nitrogen atmosphere with an externally applied magnetic field after adding $0.8 \mathrm{M} \mathrm{NaBH}_{4}$ aqueous solution. After synthesis, this notated specimen I is washed with anhydrous ethanol and dried in air. The chemical reaction mechanism (Equation 1) is shown as follows:

$$
\begin{aligned}
& 2 \mathrm{FeCl}_{3}+6 \mathrm{NaBH}_{4}+18 \mathrm{H}_{2} \mathrm{O} \rightarrow \\
& 2 \mathrm{Fe}+6 \mathrm{~B}(\mathrm{OH})_{3}+6 \mathrm{NaCl}+21 \mathrm{H}_{2}
\end{aligned}
$$

The second specimen is the same as specimen I after it undergoes hard X-ray synchrotron radiation for $10 \mathrm{~min}$ and is notated as specimen (II). The irradiation is carried out at the National Synchrotron Radiation Research Center (NSRRC) in Taiwan, with the hard X-ray beam line (BL01A). This NSRRC synchrotron light provides a $300 \mathrm{~mA}, 1.5 \mathrm{GeV}$ electron beam to generate a photon source for scientific research. The hard X-ray beam line is a semi-white-light beam line. The photon flux is $1 \times$ $10^{12}$ photons/sec, and the radiation is incident onto the sample with a beam footprint of $18.6 \times 9.3 \mathrm{~mm}^{2}$. The distribution range of photon energy is between 10 and 15 $\mathrm{keV}$, and the dose rate is $5.1 \pm 0.9 \mathrm{kGy} / \mathrm{s}$. After exposure, the specimen was washed with anhydrous ethanol and then dried.

For the third specimen, a $0.7 \mathrm{M}$ solution of iron ion was prepared by dissolving $\mathrm{FeCl}_{3} \cdot 6 \mathrm{H}_{2} \mathrm{O}$ in methanol and then mixing it with PVP and MMA monomer in a high purity nitrogen atmosphere. Then, a $0.8 \mathrm{M} \mathrm{NaBH}_{4}$ aqueous solution was dropped into the previously prepared solution to synthesize iron nanowires in an externally applied magnetic field. After forming the iron nanowires, the sample was exposed to hard X-ray synchrotron radiation for $10 \mathrm{~min}$. The obtained PMMA/Fe nanowires are denoted as specimen (III).

For the fourth specimen, a $0.7 \mathrm{M} \mathrm{FeCl}_{3} \cdot 6 \mathrm{H}_{2} \mathrm{O}$ methanol solution was mixed with PVP and MMA monomers in a high purity nitrogen atmosphere and then exposed to hard X-rays for $10 \mathrm{~min}$ to polymerize before a $0.8 \mathrm{M}$ $\mathrm{NaBH}_{4}$ aqueous solution was added under an externally applied magnetic field. The obtained sample underwent polymerization once again by hard X-ray exposure for another $10 \mathrm{~min}$.

For the fifth specimen, PMMA polymer was synthesized before iron nanowires formation. MMA stock solution was prepared by dissolving PVP (5 vol.\%) in methanol (35 vol.\%) with de-ionized water (45 vol.\%) and mixing it with MMA monomer (15 vol.\%). The stock solution was stirred in high purity nitrogen gas to eliminate oxygen. In addition, the stock solution of iron precursor was prepared in deionized water with concentrations of $0.7 \mathrm{M} \mathrm{FeCl}_{3} \cdot 6 \mathrm{H}_{2} \mathrm{O}$. Ten milliliter quantities of MMA stock solution were prepared in sealed bottles, ready for irradiation-induced polymerization for $10 \mathrm{~min}$. After that, we prepared the volume ratios of iron stock solution to PMMA emulsion solution at $1: 1$. Next, $0.8 \mathrm{M}$ $\mathrm{NaBH}_{4}$ was added to the mixture solution for self-assembled hybrid PMMA/Fe nanowires under high-purity nitrogen gas atmosphere and a magnetic field. These are called specimen $(\mathrm{V})$. The obtained sample was separated from the solution and washed with anhydrous ethanol and deionized water and then dried in air. Fe nanowires (I, II) and PMMA/Fe nanowires (III, IV, and V) were then dispersed on a carbon adhesive tape and observed by SEM. The structural properties of these specimens 
were analyzed with an X-ray diffractometer. TEM observation further confirmed the specimen's morphology. The magnetic hysteresis properties of all specimens were also analyzed by a VSM analyzer; other correlation characteristics are discussed as well.

\subsection{Material Properties}

The Fe nanowires and Fe nanowires coated with PMMA were characterized by X-ray diffraction, SEM, TEM, TGA analysis and VSM. Powder X-ray diffraction (XRD) patterns of $\mathrm{Fe}$ nanowires were obtained by employing NSRRC 17A1 W20 X-ray Powder Diffraction. An SEM HITACHI-S4700 was used to observe the morphology and shape of the Fe nanowires and hybrid PMMA/Fe nanowire materials. High resolution transmission electron microscopy (HRTEM, JEOL JEM-2100F) was used to characterize the morphology of the Fe nanowires and hybrid PMMA/Fe nanowires. The source of synchrotron radiation at the National Synchrotron Radiation Research Center (NSRRC) in Taiwan was used to study the X-ray absorption spectroscopy (XAS) of iron nanowires in the NSRRC 16A and 20A beam line. The fluorescence EXAFS technique was used in this study to detect the X-rays emitted as a result of filling the core hold created by optical absorption.

The magnetic property measurements were carried out with a VSM (Lake Shore 7407, USA) with a maximum magnetic field of $2 \mathrm{~T}$. Thermal degradation was examined with a thermal analyzer (model SDT 2960 Simultaneous DSC-TGA, TA, Germany). Each sample was weighted and filled into Pt crucibles. The samples were heated at a rate of $10^{\circ} \mathrm{C} / \mathrm{min}$ from room temperature to $1400^{\circ} \mathrm{C}$ in an oxygen flow of $20 \mathrm{~cm}^{3} / \mathrm{min}$. These measurements were conducted under oxygen flow to observe iron oxidation.

\section{Results and Discussion}

\subsection{Structural Properties}

The phase of the reduced iron nanowires has been determined from the XRD pattern as shown in Figure 1. Well defined peaks can be observed in the patterns of specimen (I) and (V), corresponding to the existence of a body centered cubic (bcc) phase of iron that coexists with $\mathrm{FeO}, \mathrm{Fe}_{3} \mathrm{O}_{4}$ and $\mathrm{Fe}_{2} \mathrm{O}_{3}$ phases in the iron nanowires As indicated in Figure 1(a), specimen (I) is composed of surface iron oxides. For the hard X-ray irradiation specimens (II) and PMMA coated specimens (III-IV), the surface layer of iron oxide gets thicker, as evidenced in Figures 1(b), (c) and (d). The results indicate that X-ray irradiation will enhance the oxidation of iron nanowires and affect the magnetic properties of these specimens.
Interestingly, after X-ray irradiation, the $\mathrm{FeO}$ and $\mathrm{Fe}_{2} \mathrm{O}_{3}$ phases disappear, and only the $\mathrm{Fe}_{3} \mathrm{O}_{4}$ phase can be observed. For specimen $(\mathrm{V})$, a pattern similar to specimen (I) can be seen, but it lacks a strong iron peak. Although the synthesis of iron nanowires occurs after X-ray irradiation, the free radicals generated by X-rays are still affecting and inducing trans-grains oxidation of iron nanowires.

\subsection{Morphology}

The morphologies of iron nanowires and hybrid PMMA/ iron nanowires, fabricated with different processing conditions, were characterized by SEM and HRTEM to compare the hard X-ray irradiation effects on them. SEM images of various iron nanowires and PMMA/Fe nanowires are shown in Figure 2. The nano wires all show bamboo-like structures. Figures 2(b), (c) and (d) show that the nanowires have parallel alignment in the same orientation, which may be due to the irradiation or hybridization of PMMA. On the other hand, Figures 2(a) and (e) show random distribution. As evidenced from the images of electron microscopy, the specimens exhibit orientation arrangement when the Fe nanowires are irradiated by hard X-ray synchrotron radiation. The diameters of both iron nanowires (I) and (II) are all about 40.1 $\pm 4.2 \mathrm{~nm}$, and the lengths of the nanowires are $584.8 \pm$ $47.2 \mathrm{~nm}$. Figures 2(c) and (d) indicate that the diameters of PMMA/Fe nanowires (III) and (IV) are about $23.3 \pm$ $3.9 \mathrm{~nm}$ and $31.5 \pm 2.5 \mathrm{~nm}$, respectively, and the lengths of these nanowires are $2.7 \pm 0.5 \mu \mathrm{m}$ and $1.1 \pm 0.2 \mu \mathrm{m}$, respectively. Nanowire (IV) undergoes polymerizations by X-ray irradiation twice, which increases the thickness

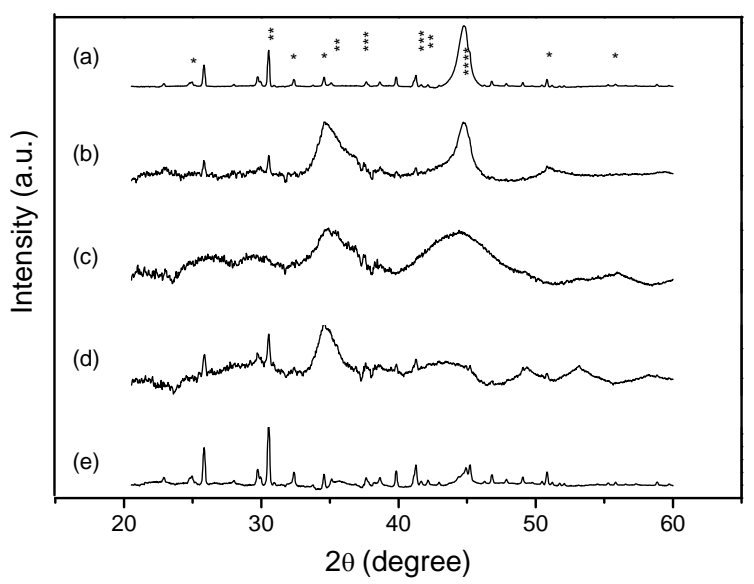

Figure 1. The XRD diffraction patterns of (a) Fe nanowires (I), (b) Fe nanowires (II) (after hard X-ray irradiation), (c) PMMA/Fe nanowires (III), (d) PMMA/Fe nanowires (IV) and (e) PMMA/Fe nanowires (V). ( ${ }^{*} \mathrm{Fe}_{2} \mathrm{O}_{3}$ peaks, ${ }^{* *} \mathrm{Fe}_{3} \mathrm{O}_{4}$ peaks, *** $\mathrm{FeO}$ peaks, and $* * * * \mathrm{Fe}$ peaks). 


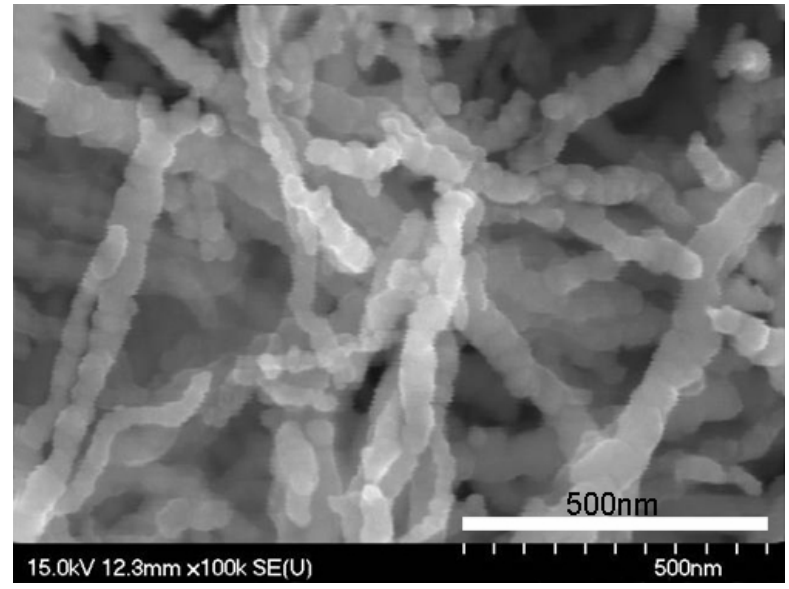

(a)

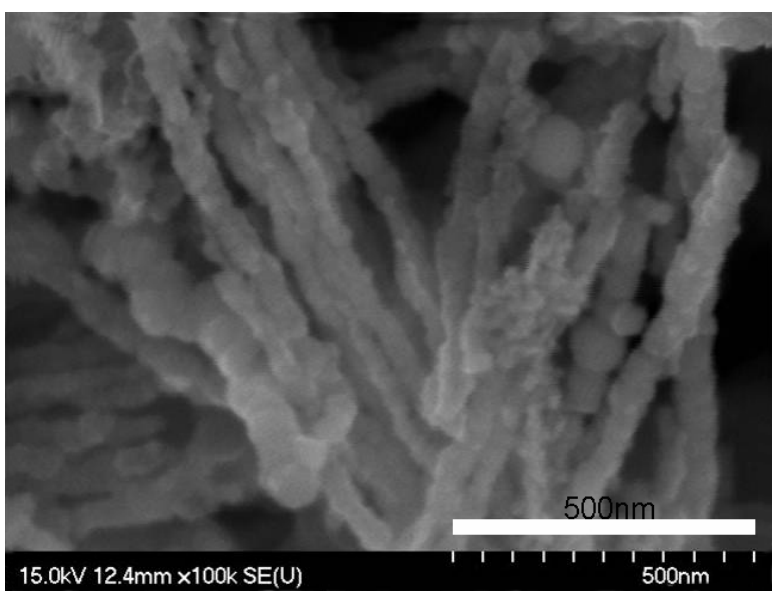

(c)

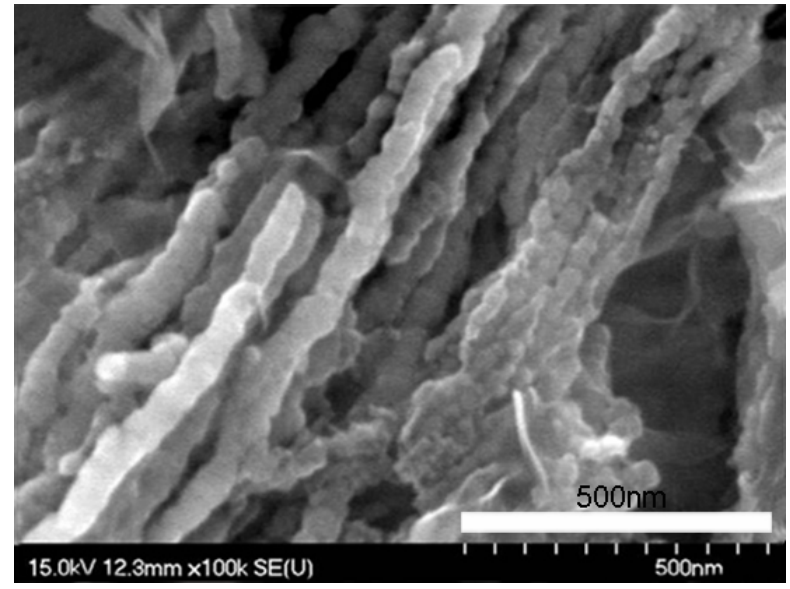

(b)

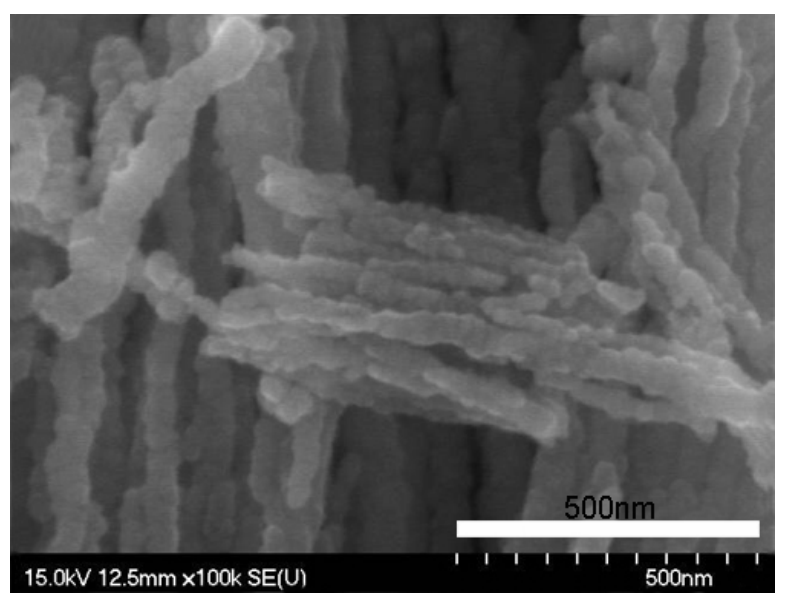

(d)

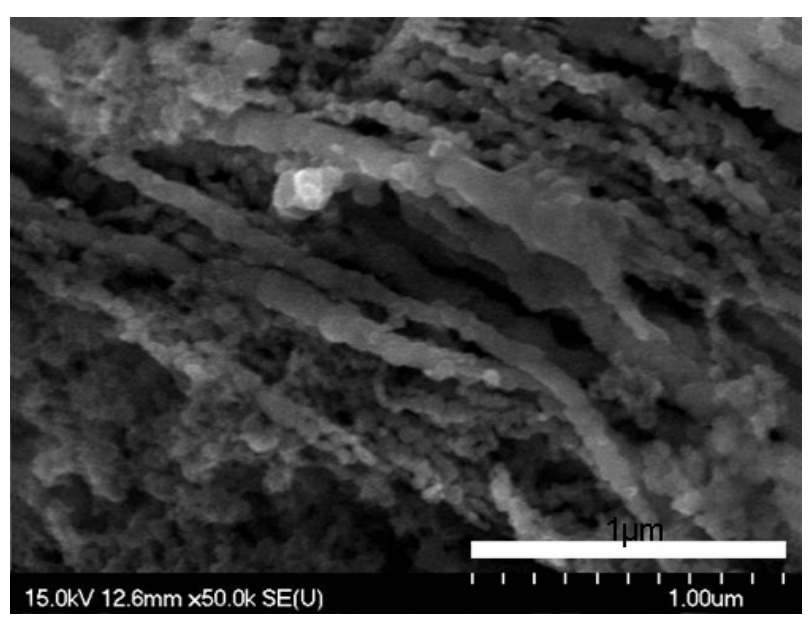

(e)

Figure 2. The SEM images of (a) Fe nanowires (I), (b) Fe nanowires (II) (after hard X-ray irradiation) (c) PMMA/Fe nanowires (III), (d) PMMA/Fe nanowires (IV) and (e) PMMA/Fe nanowires (V).

of the PMMA, but shortens the length. From the image of Figure 2(e), the morphology of PMMA/iron nanowires looks like a raspberry attached to PMMA/Fe nanoparticles and nanowires. Flocculation is observed 
between hybrid PMMA/iron nanowires. The average diameter and the average length of specimen $(\mathrm{V})$ are about $36.2 \pm 2.8 \mathrm{~nm}$ and $1.6 \pm 0.4 \mu \mathrm{m}$, respectively.

HRTEM images were used to characterize the morphologies and structures of these hybrid materials, as shown in Figure 3. For specimen (I) and (II), a thin iron

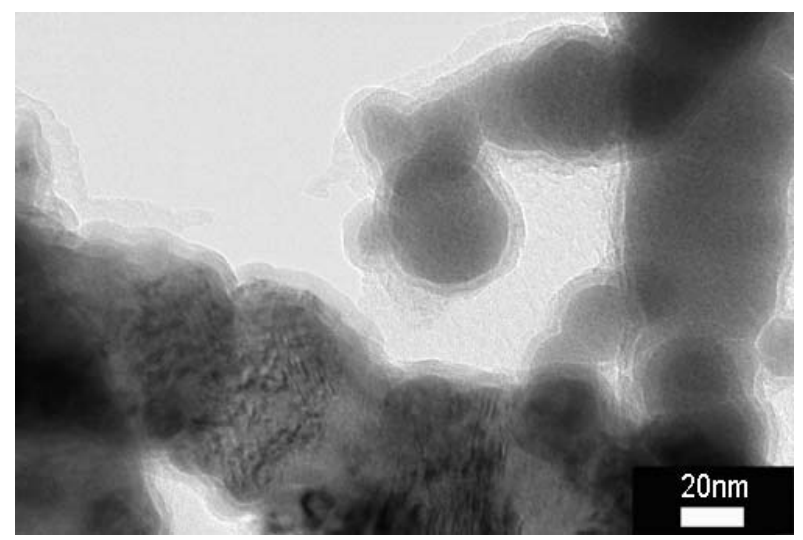

(a)

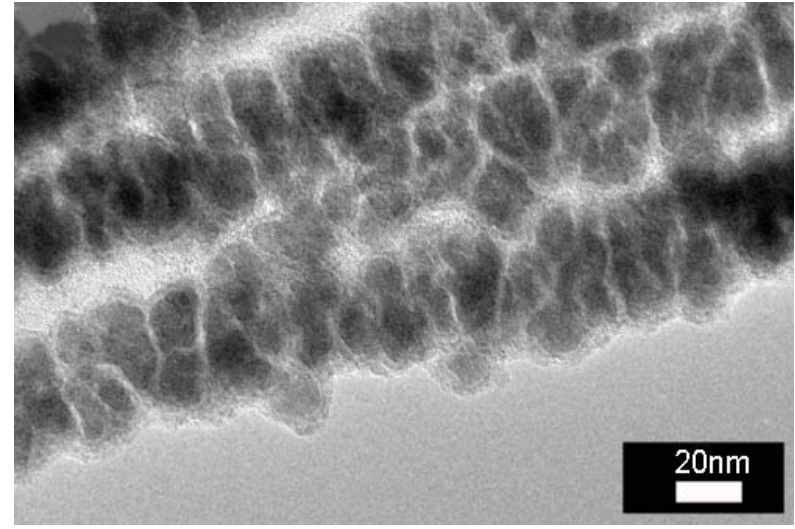

(c) oxide layer formed on the surface of the nanowires, as shown in Figures 3(a) and (b). The thickness of the iron oxide layer in specimen (I) and (II) is approximately 4.5 $\pm 0.8 \mathrm{~nm}$ and $3.5 \pm 0.2 \mathrm{~nm}$, respectively. It is important to note that for specimen (II), the X-ray irradiation induced oxidation of a thin oxide layer about $1.0 \pm 0.1 \mathrm{~nm}$

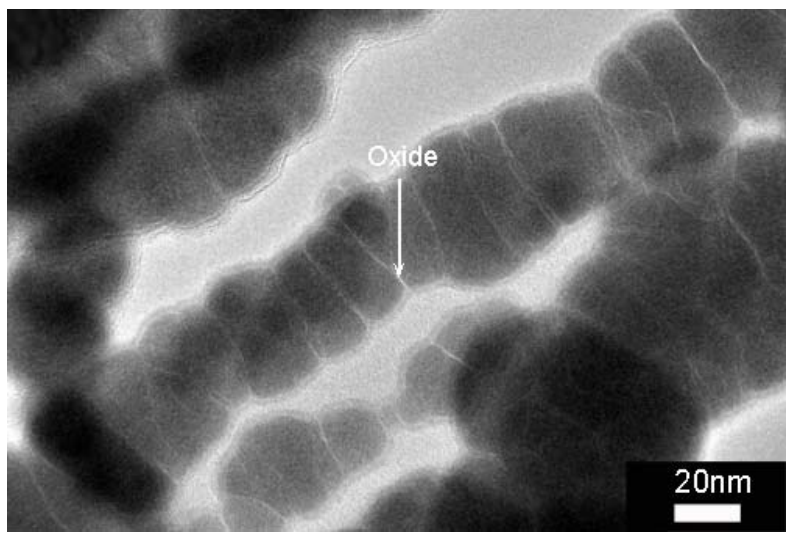

(b)

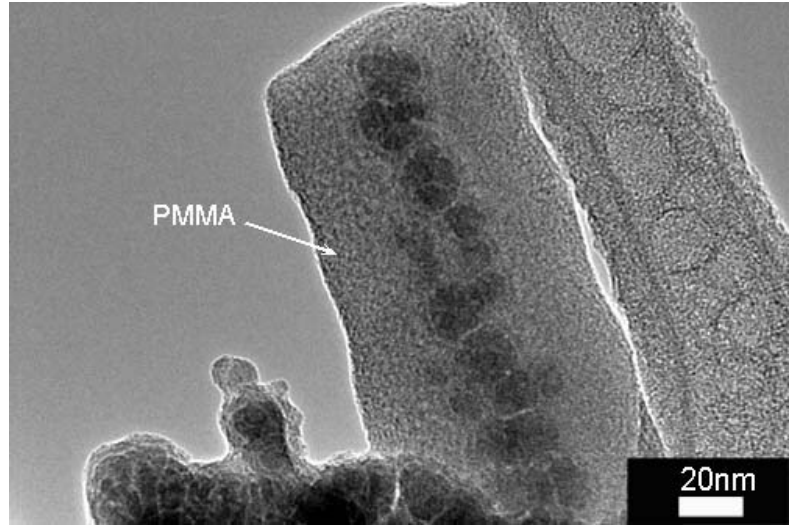

(d)

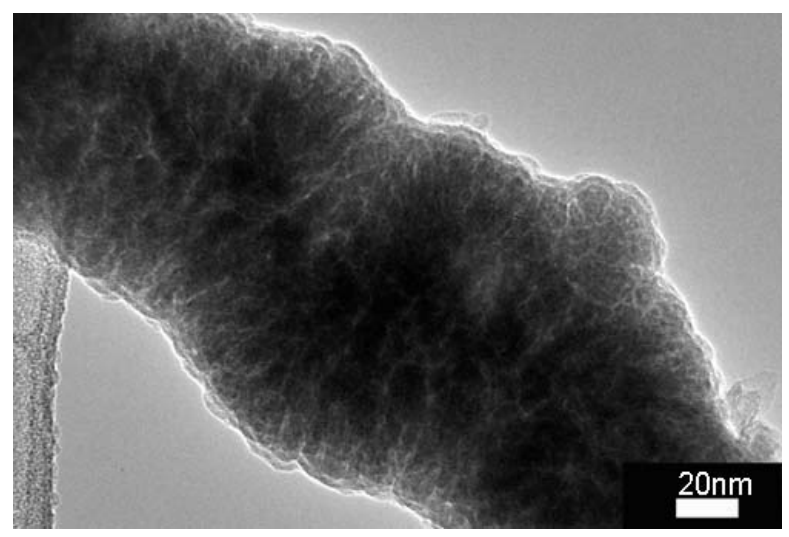

(e)

Figure 3. TEM images of Fe nanowires and PMMA/iron nanowires hybrid material with different synthesis procedures, (a) Fe nanowires (I), (b) Fe nanowires (II) (after hard X-ray irradiation), (c) PMMA/Fe nanowires (III), (d) PMMA/Fe nanowires (IV) and (e) PMMA/Fe nanowires (V). 
between the inner nanograins of iron. The diameter of the iron nanograins was measured from the TEM images in Figures 3 (a) and (b). From these, the average diameter of specimen (I) and (II) was determined to be approximately $61.7 \pm 3.8 \mathrm{~nm}$ and $40.4 \pm 11.4 \mathrm{~nm}$, respectively. Thus, X-ray irradiation not only induced oxidation of the iron inner grains, but also refined the iron grain size. This affects the properties of the iron nanowires. Figures 3(c), (d) and (e) show the coating of PMMA on the iron nanowires. The thickness of the PMMA layer is approximately $4.6 \pm 0.5 \mathrm{~nm}$ in specimen (III). In specimen (III), the layer of oxide can also be observed between iron nanograins as in specimen (II). This further proves that X-ray irradiation strongly affects the oxidation of iron between its nanograins. Specimen (IV) is not uniformly coated with PMMA, with a thickness of approximately $25.0 \pm 0.7 \mathrm{~nm}$.

In this study, iron nanowires were synthesized with or without an MMA monomer, which then underwent hard $\mathrm{X}$-ray polymerization to coat the nanowires in PMMA. Specimen (III) has a more uniform layer coating than specimen (IV). In addition, the PMMA/Fe nanowires (specimen (V)) were grown after PMMA synthesis. The thickness of the PMMA coating layer is approximately $2.6 \pm 0.1 \mathrm{~nm}$, which is smaller than specimen (III) and (IV). The X-ray diffraction patterns shown in Figure 1 indicate that the reduced iron, without a uniform PMMA protection layer, was seriously oxidized by X-ray irradiation. The thickness of the iron oxide layer in hybrid iron nanowires (III), (IV) and (V) are similar and approximately $2.9 \pm 0.3 \mathrm{~nm}, 3.3 \pm 0.5 \mathrm{~nm}$ and $2.8 \pm 0.2$ $\mathrm{nm}$, respectively. Specimen (III), (IV) and (V) have a thin oxide layer between their inner iron nanograins that were induced by hard X-ray irradiation and have thicknesses of $1.2 \pm 0.1 \mathrm{~nm}, 1.2 \pm 0.1 \mathrm{~nm}$, and $0.5 \pm 0.1$ $\mathrm{nm}$, respectively. The iron nanowires in specimens (I), (II) and (III) were all irradiated with X-rays. X-ray diffraction patterns indicate that the $\mathrm{FeO}$ and $\mathrm{Fe}_{2} \mathrm{O}_{3}$ phases disappeared in these specimens. They indicate that X-ray irradiation induced the reduction of $\mathrm{Fe}_{2} \mathrm{O}_{3}$ to form $\mathrm{Fe}_{3} \mathrm{O}_{4}$ and also caused trans-grain oxidation.

\subsection{X-ray Absorption Spectroscopy}

Iron is widely used in technologically relevant systems. The oxidation of iron surfaces has been extensively studied in the past [8]. Understanding the magnetic properties of these iron nanowires and hybrid PMMA/Iron nanowires requires a precise knowledge of the structure and composition of the oxide layer. This knowledge is important because of the different crystallographic sites of iron atoms in the oxide phases, which result in strongly structure dependent magnetic properties. X-ray absorption spectroscopy is one way to study the oxidation states and the local symmetry of atoms in solids. The X-ray absorption near-edge structure (XANES) depends directly on the oxidation states. The extended X-ray fine structure (EXAFS) includes information on the local structures [9].

The near-edge absorption spectra are acquired at the iron K-edge. The Fe K-edge spectra of the iron nanowires and hybrid PMMA/Fe nanowires samples are shown in Figure 4. The pre-edge of the spectra was used to evaluate the sensitivity of XAS to geometric and electronic structural changes [10]. Figure 4(b) shows that the iron pre-edge of iron nanowires without X-ray irradiation in specimen (I) is similar to that of commercial pure iron in Figure 4(a). It reveals that a thin oxide layer forms on the surface of as-received iron nanowires. This thin oxide layer also appears in the HRTEM image of Figure 3(a). For specimen (II) to (IV), iron nanowires are all irradiated by high intensity X-rays. They have a similar iron pre-edge as shown in Figures 4(c) to (e). The iron pre-edges of these specimens are different than that of reference $\mathrm{Fe}_{2} \mathrm{O}_{3}$ and commercial iron as shown in Figures 4(g) and (a), respectively. X-ray diffraction patterns indicate that the $\mathrm{Fe}_{3} \mathrm{O}_{4}$ phase is formed in specimen (II) to (IV). Thus, after $\mathrm{X}$-ray irradiation, the major oxide on the surface of the iron nanowires is the $\mathrm{Fe}_{3} \mathrm{O}_{4}$ phase. This also reveals that $\mathrm{X}$-ray irradiation will induce oxidation of pure iron and reduction of the $\mathrm{Fe}_{2} \mathrm{O}_{3}$ phase. Specimen $(\mathrm{V})$ is prepared with iron nanowires after forming PMMA by X-ray irradiation. Its X-ray diffraction pattern

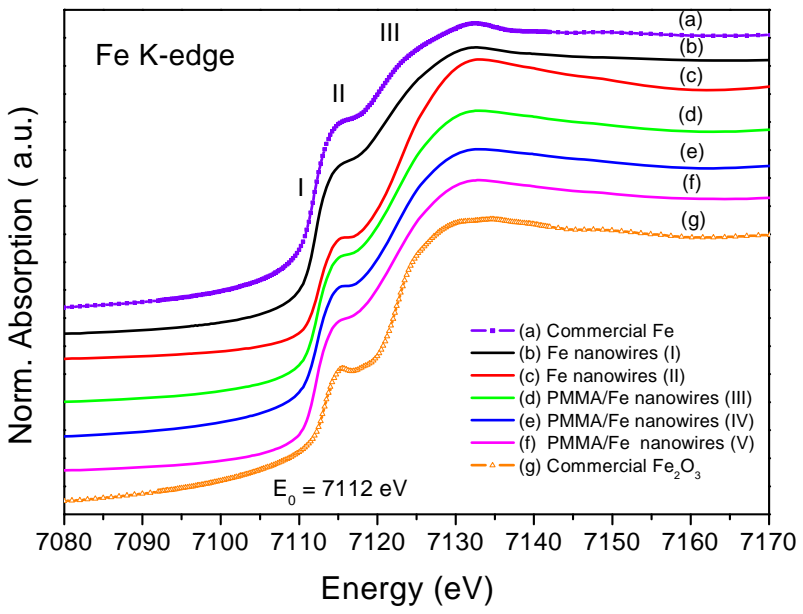

Figure 4. Fe K-edge of EXAFS of (a) commercial Fe, (b) Fe nanowires (I), (c) Fe nanowires (II) (after hard X-ray irradiation), (d) PMMA/Fe nanowires (III), (e) PMMA/Fe nanowires (IV), (f) PMMA/Fe nanowires (V), and (g) commercial $\mathrm{Fe}_{2} \mathrm{O}_{3}$. 
(Figure 1(e)) is similar to the iron nanowires without $\mathrm{X}$-ray irradiation in Figure 1(a), only with a lower intensity in the iron peak at around $2 \theta=45^{\circ}$. The shape of the iron pre-edge of specimen $(\mathrm{V})$ is similar to that of specimen (I) and specimen (II)-(IV). X-ray irradiation effects on the iron oxidephases are enhanced by the formation of $\mathrm{Fe}_{3} \mathrm{O}_{4}$ and the trans-grain oxidation, as indicated in Figures 3(b) and (c).

To further examine these results, we differentiated the data of Figure 4 to obtain the differential pre-edge of the Fe K-edge, as shown in Figure 5. The absorption edge differences in these samples are obvious. The first differential pre-edge peak represents the electron transition from the $1 \mathrm{~s}$ to $4 \mathrm{~d}$ orbital that directly relates to the oxidation state and geometry of the iron atom [11]. Also, the total intensity of this transition has been shown to in- crease with decreasing coordination number for iron due to the loss of inversion symmetry at the iron site [12]. The differential pre-edge peaks of $\mathrm{Fe}$ nanowires are listed in Table 1. The differential peak of $\mathrm{Fe}_{2} \mathrm{O}_{3}$ with $\mathrm{Fe}^{3+}$ is at $7133.3 \mathrm{eV}$, which is larger than the $7111.8 \mathrm{eV}$ of com mercial pure iron with Fe. The differential pre-edges of Iron nanowires with or without X-ray irradiation and hybrid PMMA/iron nanowires are all located between $\mathrm{Fe}_{2} \mathrm{O}_{3}$ and pure iron. For pure $\mathrm{Fe}_{2} \mathrm{O}_{3}$, there is a second differential pre-edge peak at $7117.4 \mathrm{eV}$ that is not observed in other specimens, including pure iron. It has been proven that synthesized iron nanowires have an iron oxidation state between $\mathrm{Fe}^{2+}$ and $\mathrm{Fe}^{3+}$ that forms a surface oxide with an iron core. The position of third differential pre-edge peaks is only observed as the differences between pure iron and iron nanowires (I) and the rest of the specimen. The second

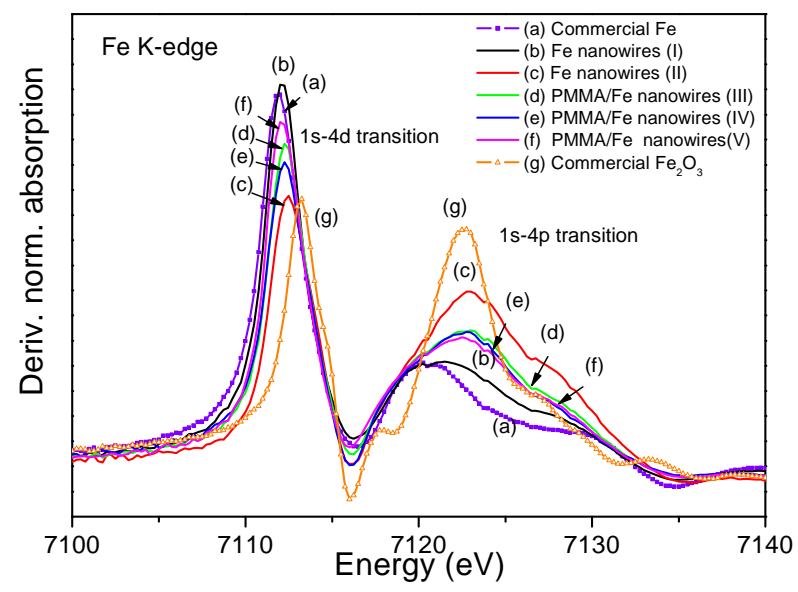

Figure 5. The differential pre-edge (NEXAFS) of the FeK-edge of (a) commercial Fe, (b) Fe nanowires (I), (c) Fe nanowires (II) (after hard X-ray irradiation), (d) PMMA/Fe nanowires (III), (e) PMMA/Fe nanowires (IV), (f) PMMA/ Fe nanowires (V), and (g) commercial $\mathrm{Fe}_{2} \mathrm{O}_{3}$. and third peaks may have been caused by the $1 \mathrm{~s}-4 \mathrm{p}$ transition [13]. It is not sensitive to different oxidation states but is between pure iron and iron oxides.

The Fourier-transformed Fe EXAFS data in Figure 6 indicate that the nearest Fe-O bond appears at about 1.4$1.6 \AA$ for all specimens, corresponding to a tetrahedrally coordinated cation. For commercial iron and iron nanowires without $\mathrm{X}$-ray irradiation, the $\mathrm{Fe}-\mathrm{Fe}$ bond length of metallic iron is about $2.1 \AA$ and can be observed, but not in other specimens. XAS, in this study, was measured in fluorescence mode. Specimens (II) to (V) have a thicker oxide layer compared to specimen (I). Thus, the Fe-Fe bond is not observed in these specimens. The radiation distribution of specimen (II) to (V) shows major differences compared to commercial $\mathrm{Fe}_{2} \mathrm{O}_{3}$. A previous study [14] indicated that the oxide layer in these nanowires is $\mathrm{Fe}_{3} \mathrm{O}_{4}$. XANES of the $\mathrm{O} \mathrm{K}$ edge spectra in Figure 7 also shows dramatic differences between $\mathrm{Fe}_{2} \mathrm{O}_{3}$ and the rest of specimens. In the $\mathrm{O} \mathrm{K}$-edge, split absorption peaks are observed only in $\mathrm{Fe}_{2} \mathrm{O}_{3}$. This shows that the iron nanowires can be stabilized by the $\mathrm{Fe}_{3} \mathrm{O}_{4}$ surface oxide layer.

As seen in the HRTEM images of Figure 3, transgrain oxidation occurs when X-rays irradiate iron nanowires. XAS and XRD results indicate that the oxide phase after $\mathrm{X}$-ray irradiation is mainly $\mathrm{Fe}_{3} \mathrm{O}_{4}$. The oxide layer also got larger after irradiation. The mechanism of X-ray induced oxidation may be the following;

$$
\begin{gathered}
2 \mathrm{Fe}_{(\text {core })}+\mathrm{O}_{2} \stackrel{h v}{\longrightarrow} 2 \mathrm{FeO} \\
\mathrm{FeO}+\mathrm{Fe}_{2} \mathrm{O}_{3(\text { surface })} \stackrel{h v}{\longrightarrow} \mathrm{Fe}_{3} \mathrm{O}_{4(\text { surface })}
\end{gathered}
$$

The iron atoms diffuse out from the core of the nanoparticles that form the iron nanowires. Under X-ray irradiation, iron atoms first oxidize into $\mathrm{FeO}$ (Equation 2) and then react with $\mathrm{Fe}_{2} \mathrm{O}_{3}$ to form $\mathrm{Fe}_{3} \mathrm{O}_{4}$ (Equation 3). Thus, the trace $\mathrm{Fe}_{2} \mathrm{O}_{3}$ phase in specimen (I) is transferred into the $\mathrm{Fe}_{3} \mathrm{O}_{4}$ phase, a fact that is proven by XRD and XAS results.

Table 1. The differential pre-edge peaks of Fe nanowires and PMMA/Fe nanowires.

\begin{tabular}{lccc}
\hline \multicolumn{1}{c}{ Specimens } & First (eV) & Second (eV) & Third (eV) \\
\hline Fe nanowires (I) & 7112.1 & - & 7120.3 \\
Fe nanowires (II) & 7112.4 & - & 7122.7 \\
PMMA/Fe nanowires (III) & 7112.2 & - & 7122.7 \\
PMMA/Fe nanowires (IV) & 7112.2 & - & 7122.7 \\
PMMA/Fe nanowires (V) & 7112.1 & - & 7122.7 \\
Commercial Fe $\mathrm{O}_{3}$ & 7113.3 & 7117.4 & 7122.7 \\
Commercial Fe & 7111.8 & - & 7120.3 \\
\hline
\end{tabular}




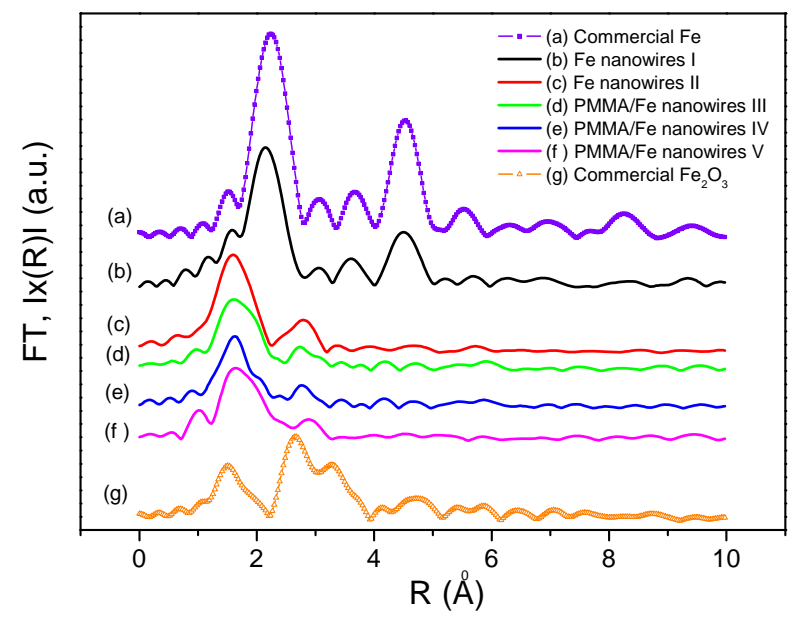

Figure 6. Fourier-transformed Fe EXAFS data of (a) commercial Fe, (b) Fe nanowires (I), (c) Fe nanowires (II) (after hard X-ray irradiation), (d) PMMA/Fe nanowires (III), (e) PMMA/Fe nanowires (IV), (f) PMMA/Fe nanowires (V), and (g) commercial $\mathrm{Fe}_{2} \mathrm{O}_{3}$.

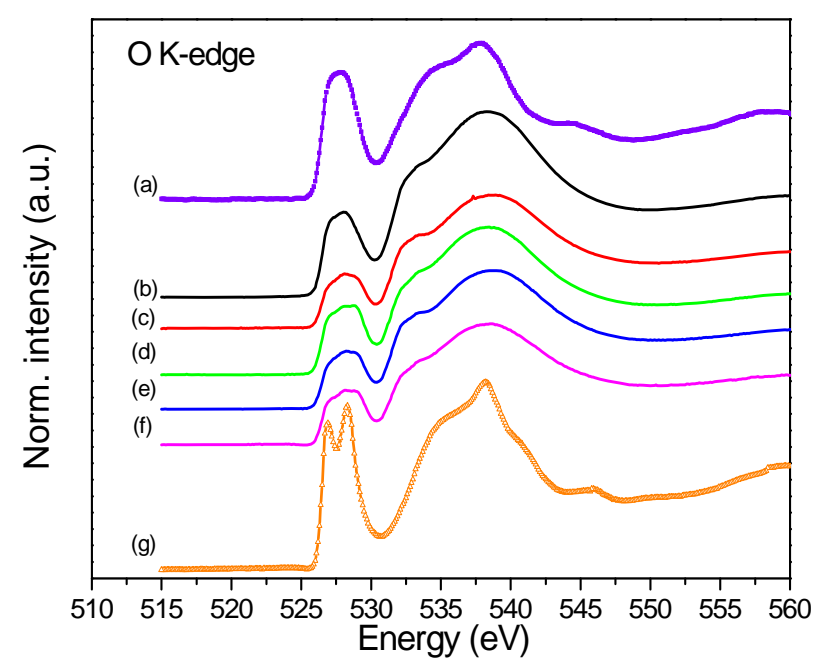

Figure 7. XANES normalized intensity of the $\mathrm{O} K$ edge spec tra of (a) commercial $\mathrm{Fe}$, (b) Fe nanowires (I), (c) Fe nanowires (II) (after hard X-ray irradiation), (d) PMMA/Fe nanowires (III), (e) PMMA/Fe nanowires (IV), (f) PMMA/Fe nanowires (V), and (g) commercial Fe2O3.

\subsection{TGA Analysis}

To measure the mass content of the specimens, the iron nanowires and the hybrid PMMA/Fe nanowires were examined by TGA analysis with an SDT2960 Simultaneous DSC-TGA (TA Instruments) system. The results are shown in Figure 8. The curves show mass decreases in specimen (I), (II), (III), (IV) and (V) prior to 166 , 303, 216, 254 and $324^{\circ} \mathrm{C}$, respectively, under oxygen atmosphere. Specimen (I), (II), (III), (IV) and (V), had around

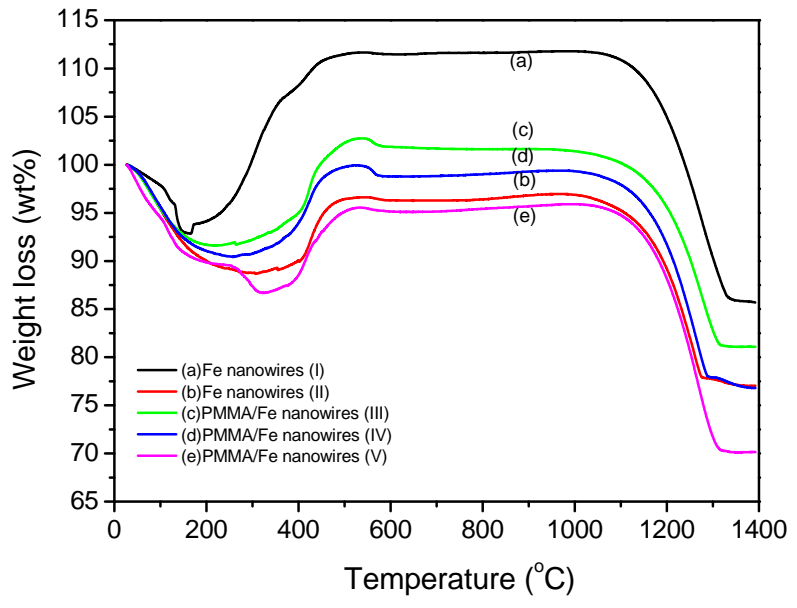

Figure 8. The TGA spectrums of (a) Fe nanowires (I), (b) Fe nanowires (II) (after hard X-ray irradiation), (c) PMMA/Fe nanowires (III), (d) PMMA/Fe nanowires (IV) and (e) PMMA/Fe nanowires (V).

$7.18,11.29,8.48,9.56$, and $13.31 \mathrm{wt} \%$ mass loss, respectively, including water, methanol and thermal decomposition of hydroxide materials. Then, the iron nanowires started to oxidize and PMMA began to decompose. After PMMA completely decomposed, the weights of the specimens were greater than $100 \%$, due to iron oxide formation. Specimens (III), (IV) and (V) have a peak at around $500^{\circ} \mathrm{C}$ that may have been cause by the carbonization of PMMA. The Curve (a) for specimen (I) in Figure 8 shows that the oxidation temperature of iron nanowires is lower than that of other specimens. This indicates that the iron nanowires without X-ray or PMMA treatments are oxidized more easily than other specimens. This shows that the inner nanograins of specimen (II) to (V) are protected by thin layers of oxides that resist oxidation at lower temperatures. The weight gain of specimen (I) due to oxidation is great larger than that of specimen (II). This indicates that the iron nanowires treated by X-ray irradiation are highly oxidized. This phenomenon is also proven by the $\mathrm{X}$-ray dif- fraction pattern in Figure 1. The major diffraction peak of pure iron in specimen (II) is from dramatic reduction. Specimens (III) to (V) show the same phenomenon as specimen (II). Pure PMMA usually decomposes before $430^{\circ} \mathrm{C}$, but the weight gain peak decreased in specimens (III), (IV) and (V) when the temperature was higher than $600^{\circ} \mathrm{C}$. This may be due to further decomposition of the residues of carbonized PMMA. The iron oxides were reduced at temperatures higher than $1100^{\circ} \mathrm{C}$, which causes weight loss in the TGA thermogram. At temperatures $>1350{ }^{\circ} \mathrm{C}$, the 
residual weight of the specimens indicates their iron content. The residue weight of specimen (II) at $1350^{\circ} \mathrm{C}$ is $77.0 \mathrm{wt} \%$, less than that of specimen (I), which is about $85.8 \mathrm{wt} \%$, as shown in Figure 8. This confirms that X-ray irradiation induced the oxidation of specimen (II). Thus, the oxide content in specimen (II) is larger than in specimen (I). According to residual weight in the TGA thermogram, the iron contents of specimens (I) to (V) are about $85.8,77.0,81.2,76.7$ and $70.1 \mathrm{wt} \%$, respectively.

\subsection{Magnetic Properties}

It was recently shown that nanometer thick native-oxide layers can be used as building blocks in new magnetic structures [15]. For multilayers consisting of alternating $\mathrm{Fe}$ and Fe-oxide layers, the magnetic moments of the Fe layers are arranged in a non-collinear fashion [16]. The structure of nanowires after X-ray irradiation can build a chain of iron nanoparticle/iron nano oxide layer structures. This may lead to novel magnetic materials for application in the electronics or medical fields.

The magnetic properties of the various specimens are examined at room temperature by VSM. Figure 9 and Table 2 show magnetic hysteresis curves and data of these specimens, respectively. The hysteresis loops of specimens (I), (II), (III), (IV) and (V) reveal their ferromagnetic behavior. Specimen (II), with X-ray irradiation, has a saturation magnetization (Ms) that is $173 \mathrm{emu} / \mathrm{g}$ larger than that of specimen (I) $(138 \mathrm{emu} / \mathrm{g})$. Their remanences (Mr) are 64 and $51 \mathrm{emu} / \mathrm{g}$, respectively. TEM images of Figure 3 (a) and (b) show that the diameter of iron nanograins is approximately $40.4 \pm 11.4$ $\mathrm{nm}$ for specimen (II) and $61.7 \pm 3.8 \mathrm{~nm}$ for specimen (I). These results demonstrate that hard X-ray irradiation causes the refinement of grain size and also induces oxidation reactions in iron nanograins. For specimens (I) and (II), the coercivity (Hc) is 789 Oe (reduced remanence ratio 0.37) and 324 Oe (reduced remanence ratio 0.37 ), respectively. The saturation magnetization of PMMA/Fe nanowires (III, IV and V) is 101, 82 and 71 $\mathrm{emu} / \mathrm{g}$; the coercivity is 171,116 and $60 \mathrm{Oe}$; and remanence is 39,31 and $13 \mathrm{emu} / \mathrm{g}$, respectively.

The magnetic properties of these specimens are attributed to the size and structure of the iron and iron oxides. For specimens (I) and (II), it is clear that X-ray irradiation induces size refinement and reduction of iron oxide. The $\mathrm{Fe}_{2} \mathrm{O}_{3}$ phase is reduced to $\mathrm{Fe}_{3} \mathrm{O}_{4}$. This is not only the case in specimen (II). Specimens (III) and (IV) are also only reduced to $\mathrm{Fe}_{3} \mathrm{O}_{4}$ as observed in X-ray diffraction patterns. These nanowires are synthesized under strong magnetic fields, which arranged the nanograins in magnetically preferred orientation.

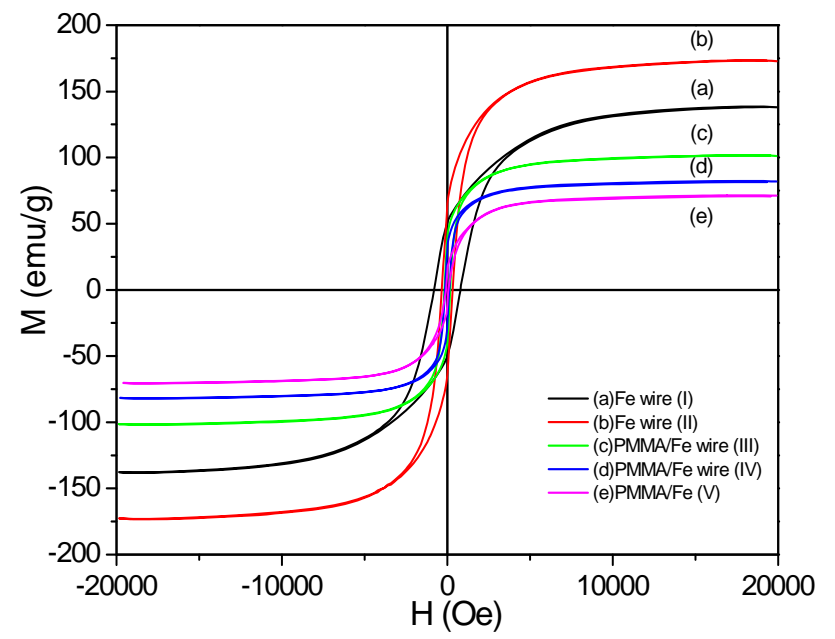

Figure 9. The VSM measurements of (a) Fe nanowires (I), (b) Fe nanowires (II) (after hard X-ray irradiation), (c) PMMA/Fe nanowires (III), (d) PMMA/Fe nanowires (IV) and (e) PMMA/Fe nanowires (V).

Table 2. The VSM measured data of Fe nanowires and PMMA/Fe nanowires.

\begin{tabular}{lcccc}
\hline \multicolumn{1}{c}{ Specimens } & $\begin{array}{c}\mathrm{Hc} \\
(\mathrm{Oe})\end{array}$ & $\begin{array}{c}\mathrm{Mr} \\
(\mathrm{emu} / \mathrm{g})\end{array}$ & $\begin{array}{c}\mathrm{Ms} \\
(\mathrm{emu} / \mathrm{g})\end{array}$ & $\mathrm{Sr}=\mathrm{Mr} / \mathrm{Ms}$ \\
\hline Fe nanowires (I) & 789 & 51 & 138 & 0.37 \\
Fe nanowires (II) & 324 & 64 & 173 & 0.37 \\
PMMA/Fe nanowires (III) & 171 & 39 & 101 & 0.39 \\
PMMA/Fe nanowires (IV) & 116 & 31 & 82 & 0.38 \\
PMMA/Fe nanowires (V) & 60 & 13 & 71 & 0.18 \\
\hline
\end{tabular}

The magnetic saturation of iron nanowires and PMMA/iron nanowires is higher than that of the spherecal Fe colloids produced by sonochemical synthesis (101 $\mathrm{emu} / \mathrm{g}$ ) [17] or iron nanoparticles produced by metal vapor deposition $(55 \mathrm{emu} / \mathrm{g})$ [18]. Figure 1 shows the effect of the remaining $\mathrm{Fe}_{2} \mathrm{O}_{3}$ and $\mathrm{Fe}_{3} \mathrm{O}_{4}$ phases. Both remanence and coercivity are affected by the volume fraction of the $\mathrm{Fe}_{3} \mathrm{O}_{4}$ phase. The magnetic properties of iron nanowires are similar to nanocrystalline soft materials where the magnetization averages over several grains [19]. The exchange interactions between iron nanograins in nanowires due to agglomeration account for the remanence enhancement and the drop of the coercive field as shown in specimen (II) with respect to the Stoner-Wohlfarth value [20].

\section{Conclusions}

In summary, iron nanowires and hybrid PMMA/Fe nanowires were successfully synthesized with the 
reducing agent $\mathrm{NaBH}_{4}$ under an externally applied magnetic field with radiation polymerization. The externally applied magnetic field played an important role in the formation of uniform and parallel iron nanowires.

The bamboo-like structure of the iron nanowires could be observed by SEM and HRTEM. An oxide layer formed on the surface of the iron nanowires. XRD results showed that the $\mathrm{Fe}_{2} \mathrm{O}_{3}$ and $\mathrm{Fe}_{3} \mathrm{O}_{4}$ phases coexisted in the iron nanowires without $\mathrm{X}$-ray irradiation. When these samples are irradiated by hard X-ray from NSRRC, $\mathrm{Fe}_{3} \mathrm{O}_{4}$ is the only phase that can be observed. The Fe and $\mathrm{O}$ K-edges of XAS were used to distinguish the oxide structures on the surface of the iron nanowires and PMMA/iron nanowires. The results of XAS show the phase transformation of $\mathrm{Fe}_{2} \mathrm{O}_{3}$ to $\mathrm{Fe}_{3} \mathrm{O}_{4}$. Thus, X-ray irradiation will induce $\mathrm{Fe}_{2} \mathrm{O}_{3}$ phase transformation to $\mathrm{Fe}_{3} \mathrm{O}_{4}$ and stabilize these iron nanowires. TGA analysis is used here to determine the thermal properties and solid content of these specimens.

Magnetic hysteresis measurements reveal that the iron nanowires displayed ferromagnetic behavior. For the iron nanowires (specimen (II)) prepared after X-ray irradiation, the saturation magnetization is $173(\mathrm{emu} / \mathrm{g})$ larger than that of the initial iron nanowires (specimen (I)). The saturation magnetization of the iron nanowires and PMMA/iron nanowires is higher than that of spherical Fe colloids produced by sonochemical synthesis or iron nanoparticles produced by metal vapor deposition. Iron nanowires-coated PMMA hybrid materials could be designed to manipulate the magnetic and biocompatibility properties for application in magnetic hyperthermia. This study indicates that X-ray irradiation treatment of these iron nanowires will provide a way to manipulate the structural and magnetic properties of novel hybrid nano-materials.

\section{Acknowledgements}

The authors gratefully acknowledge the National Science Council, Taiwan, for financial support, and the TLS (01A) team of the National Synchrotron Radiation Research Center (NSRRC) for technical support.

\section{REFERENCES}

[1] L. Menon, M. Zheng, H. Zeng, S. Bandyopadhyay and D. J. Sellmyer, "Size Dependence of the Magnetic Properties of Electrochemically Self-Assembled Fe Quantum Dots," Journal of Electronic Materials, Vol. 29, No. 5, pp. 510515.

[2] S. Bandyopadhyay, L. Menon, N. Kouklin, H. Zeng and D. J. Sellmyer, "Electrochemically Self-Assembled Quantum Dot Arrays," Journal of Electronic Materials,
Vol. 28, No. 5, 2000, pp. 515-519.

[3] M. Zheng, L. Menon, H. Zeng, Y. Liu, S. Bandyopadhyay, R. D. Kirby and D. J. Sellmyer, "Magnetic Properties of Ni Nanowires in Self-Assembled Arrays," Physical Review B, Vol. 62, No. 18, 2000, pp. 1228212286.

[4] S. Pan, Z. An, J. Zhang, and G. Song, "Facile Synthesis of Prickly CoNi Microwires," Materials Letters, Vol. 64, No. 3, 2010, pp. 453-456.

[5] P. Liu, Z. Li, B. Zhao, B. Yadian, B. and Y. Zhang, "Template-Free Synthesis of Nickel Nanowires by Magnetic Field," Materials Letters, Vol. 63, No. 20, 2009, pp. 1650-1652.

[6] H. Liou, W. Liou, H. Lin, Y. Hwu and W. Chen, "Characterization of Gold/PMMA Hybrid Nanomaterials Synthesized by Hard X-Ray Synchrotron Radiation," Particuology, Vol. 8, No. 3, pp. 234-239.

[7] H. M. Lin, C. K. Lin and Y. K. Hwu, "X-Ray Absorption Techniques in Examination the Structural Properties of Nanocrystalline Materials," Fu Jen Studies, Science and Engineering, Vol. 38, 2004, pp. 1-26.

[8] S. J. Roosendaal, A. M. Vredenberg and F. H. P. M. Habraken, "Oxidation of Iron: The Relation between Oxidation Kinetics and Oxide Electronic Structure," Physical Review Letters, Vol. 84, No. 15, 2000, pp. 3366-3369.

[9] S. Couet, T. H. Diederich, K. Schlage and R. Röhlsberger, "A Compact UHV Deposition System for in situ Study of Ultrathin Films via Hard X-Ray Scattering and Spectroscopy," Review of Scientific Instruments, Vol. 79, No. 9, 2008, pp. 093908-1-093908-9.

[10] G. Giuli, G. Pratesi, C. Ciprianiand and E. Paris, "Iron Local Structure in Tektites and Impact Glasses by Extended X-Ray Absorption Fine Structure and HighResolution X-Ray Absorption Near-Edge Structure Spectroscopy," Geochimica et Cosmochimica Acta, Vol. 66, No. 24, 2002, pp. 4347-4353.

[11] R. G. Shulman, Y. Yafet, P. Eisenberger and W. E. Blumberg, "Observation and Interpretation of X-Ray Absorption Edges in Iron Compounds and Proteins," Proceedings of the National Academy of Sciences of the United States of America, Vol. 73, No. 5, 1976, pp. 1384-1388.

[12] C. R. Randall, L. Shu, Y. M. Chiou, K. S. Hagen, M. Ito, N. Kitajima, R. J. Lachicotte, Y. Zang and L. Jr. Que, "X-Ray Absorption Pre-Edge Studies of High-Spin Iron (II) Complexes," Inorganic Chemistry, Vol. 34, No. 5, 1995, pp. 1036-1039.

[13] T. E. Westre, P. Kennepohl, J. G. DeWitt, B. Hedman, K. O. Hodgson and E. I. Solomon, "A Multiplet Analysis of Fe K-Edge 1s $\rightarrow$ 3d Pre-Edge Features of Iron Complexes," Journal of The America Chemical Society, Vol. 119, No. 27, 1997, pp. 6297-6314.

[14] S. Son, M. Taheri, E. Carpenter, V. G. Harris and M. E. McHenry, "Synthesis of Ferrite and Nickel Ferrite Nanoparticles Using Radio-Frequency Thermal Plasma Torch," Journal of Applied Physics, Vol. 91, No. 10, 2002, pp. 
7589-7591.

[15] G. S. D. Beach, F. T. Parker, D. J. Smith, P. A. Crozier and A. E. Berkowitz, "New Magnetic Order in Buried Native Iron Oxide Layers," Physical Review Letters, Vol. 91, No. 26, 2003, pp. 267201-1-267201-4.

[16] T. H. Diederich, S. Couet and R. Röhlsberger, "Noncollinear Coupling of Iron Layers through Native Iron Oxide Spacers," Physical Review B, Vol. 76, No. 5, 2007, pp. 054401-1-054401-5.

[17] K. S. Suslick, M. Fang and T. Hyeon, "Sonochemical Synthesis of Iron Colloids," Journal of the America Chemical Society, Vol. 118, No. 47, 1996, pp. 11960-11961.

[18] C. F. Kernizan, K. J. Klabunde, C. M. Sorensen and G. C.
Hadjipanayis, "Magnetic Properties of Nanometer-Scale Iron Particles Generated by Iron Atom Clustering in Cold Pentane," Chemistry of Materials, Vol. 2, No. 1, 1990, pp. 70-74.

[19] W. J. M. Mulder, G. J. Strijkers, G. A. F. van Tilborg, A, W. Griffioen and K. Nicolay, "Lipid-Based Nanoparticles for Contrast-Enhanced MRI and Molecular Imaging," NMR in Biomedicine, Vol. 19, No. 1, 2006, pp. 142-164.

[20] E. V. Shtykova, X. Huang, N. Remmes, D. Baxter, B. Stein, B. Dragnea, D. I. Svergun and L. M. Bronstein, "Structure and Properties of Iron Oxide Nanoparticles Encapsulated by Phospholipids with Poly(ethylene glycol) Tails," The Journal of Physical Chemistry C, Vol. 111, No. 49, 2007, pp. 18078-18086. 